\title{
Treatment of acute otitis externa with ciprofloxacin otic $0.2 \%$ antibiotic ear solution
}

\author{
This article was published in the following Dove Press journal: \\ Therapeutics and Clinical Risk Management \\ 26 July $201 \mathrm{I}$ \\ Number of times this article has been viewed
}

\author{
R Mösges \\ M Nematian-Samani \\ A Eichel \\ Institute of Medical Statistics, \\ Informatics and Epidemiology, \\ Faculty of Medicine, University \\ of Cologne, Germany
}

Correspondence: Ralph Mösges Institute of Medical Statistics, Informatics and Epidemiology, Faculty of Medicine, University of Cologne, Lindenburger Allee 42, 5093। Cologne, Germany Tel +49 22I 4783456 Fax +49 22I 4783465 Email ralph@moesges.de
Background/objective: An inflammation of the cutis and subcutis of the external auditory canal is a primary symptom in cases of acute otitis externa. It is usually treated locally, since this type of therapy ensures a high concentration of the drug and interacts at the site of inflammation with no systemic effects. This systematic review compares the efficacy of treatment using a ciprofloxacin $0.2 \%$ solution with other therapeutic options.

Methods: After compiling a catalog of search terms, medical databases were searched systematically for randomized, controlled studies. This search initially yielded a total of 38 studies which were then evaluated by three independent reviewers. The number of studies was subsequently reduced to 14 : six studies using a ciprofloxacin $0.2 \%$ solution, and eight studies using both $0.2 \%$ and $0.3 \%$ solutions.

Results: The studies included in the review demonstrate the statistical equivalence between the ciprofloxacin solution $(0.2 \%$ ) and the reference products $\mathrm{PNH}$ (a combination of polymyxin B, neomycin sulfate and hydrocortisone), auriculum powder, and a ciprofloxacin foam with respect to the cure rate. The research groups consistently observed high in vitro activity of ciprofloxacin against Pseudomonas aeruginosa.

Conclusion: This systematic review confirms the hypothesis of ciprofloxacin's noninferiority in the treatment of otitis externa, in terms of the cure rate and microbiological eradication.

Keywords: otitis externa, ciprofloxacin, antibiotic, ear solution, efficacy

\section{Introduction}

\section{Otitis externa}

Inflammation of the cutis and subcutis of the external auditory canal is a primary symptom in acute otitis externa. An affected pinna can be a secondary symptom. Occasionally, the eardrum can also be inflamed. ${ }^{1}$ Inflammation of the ear can occur in an acute and a chronic form. In some cases, the clinical picture develops to a necrotizing stage. Statistically, one in ten people suffers at least once in his life from otitis externa. In $10 \%$ of cases, the inflammation is bilateral. ${ }^{2}$ Currently, many different therapies are applied to ease the symptoms. The purpose of this review is to compare the efficacy of ciprofloxacin $0.2 \%$ antibiotic ear solution with other treatment options.

\section{General etiology}

An intact auditory canal possesses the ability to cleanse itself by migrating the sloughed epithelia cells outwards with cerumen. The main function of cerumen is to protect the membrane that lines the auditory canal against inflammation. Cerumen maintains the soft consistency of the membrane and also ensures water resistance. Whether it also 
has an antimicrobial effect has not yet been clarified. If the cerumen is pushed from the outer part of the auditory canal toward the eardrum using a cotton swab, its effectiveness is lowered. Should bacteria then enter the ear canal, the risk of progressive bacterial growth increases. This can occur particularly in swimming pools, which is why the term "swimmer's ear" is commonly used. ${ }^{3}$

Likewise, congenital or acquired anatomical anomalies (eg, narrow passages), the use of hearing aids, or the aforementioned radical ear and ear canal hygiene with the complete removal of cerumen or drainage can destabilize the sensitive environment and thus predispose the external auditory canal to inflammation. ${ }^{1}$

\section{Pathogens}

The $\mathrm{pH}$ of the external auditory canal varies between 5.0 and 5.7 and is therefore slightly acidic. Such conditions inhibit bacterial growth. ${ }^{1}$ In 1981, Brook examined the physiological, normal flora of the external auditory canal in pediatric patients. In descending order of concentration, colonization with aerobes such as Staphylococcus epidermidis, diphtheroid species, and a-hemolyzing streptococci as well as anaerobes such as propionibacterium acnes, was observed. Pseudomonas aeruginosa and Staphylococcus aureus act pathogenically against such flora and are cited in the technical literature as the main causative organisms. Sporadically, viruses and fungi can also cause otitis externa. ${ }^{1,4}$

\section{Clinical picture}

Bacterial otitis externa in its mild form can be accompanied by only minor pain and subdued swelling. In its severe form, however, the symptoms are associated with excruciating pain, otorrhea, and the complete closure of the external auditory canal. The result is conductive deafness. ${ }^{1}$

Apart from the typical acute form of otitis externa, special forms can appear such as otitis externa circumscripta, which emanates from a hair follicle inflammation, or otitis externa necroticans ("maligna"), which can take a fulminant course and therefore requires maximum, usually intravenous treatment. ${ }^{1,5}$

In the majority of published clinical studies on the treatment of otitis externa, pain, swelling, otorrhea, and redness are evaluated as typical parameters for rating the clinical signs.

\section{Therapy}

Otitis externa is usually treated locally. ${ }^{1}$ Ototoxic antibiotics such as aminoglycosides should not be applied in patients with a perforated tympanic membrane. If an antibiogram has been made, the optimum antibiotic otologic drug can be determined. If none is available, "calculated antibiosis" is recommended, ie, a drug is used that is effective against the two most common pathogens $S$. aureus and $P$. aeruginosa. ${ }^{6}$ Individual decisions must be made in case of resistance. Often, an antiseptic ingredient such as aluminium acetate/ acetic acid is added to the antibiotic. Due to their acidic properties, these substances are especially suitable for lowering the $\mathrm{pH}$ value in the auditory canal, so that the main pathogens $P$. aeruginosa and $S$. aureus, which reach their optimal $\mathrm{pH}$ between 6.5 and 7.3, do not obtain perfect growing conditions or, in an ideal case, are killed.,

For years, glucosteroids had the reputation of primarily reducing the swelling of the auditory canal. Newer studies, however, also ascribe to them antibacterial and antifungal effects in otitis externa. Yet the number of available studies on steroidal monotherapies is still rather low. ${ }^{5}$

Nonsteroidal anti-inflammatory drugs should also be administered for pain relief. ${ }^{1}$

\section{Ciprofloxacin}

Ciprofloxacin is a synthetic antibiotic with a broad spectrum of activity and has the chemical formula $\mathrm{C}_{17} \mathrm{H}_{18} \mathrm{FN}_{3} \mathrm{O}_{3}$. Belonging to the group of fluoroquinolones (gyrase inhibitors), it acts as a bactericide particularly against gram-negative pathogens by inhibiting DNA replication (topoisomerase II) and interfering in the enzymatic activity of topoisomerase IV, both of which are required for the bacteria's cell division, transcription, repair, and recombination. It is moderately effective against grampositive pathogens, while it shows no relevant activity against fungi or parasites. ${ }^{8}$

In $75 \%$ of cases, ciprofloxacin is eliminated unchanged by renal excretion. It is also metabolized through the liver and eliminated through bile and is thus subject to enterohepatic circulation. ${ }^{8}$ Ciprofloxacin ranks among the most effective fluoroquinolones against $P$. aeruginosa and can also show very high in vitro activity against enterobacteria and Haemophilus influenzae. Being the only antibiotic available for oral treatment of infections caused by $P$. aeruginosa, it is administered in particular to treat chronic purulent otitis media, and can be applied locally and systemically to treat acute otitis externa. ${ }^{9}$

Ciprofloxacin constitutes the drug of choice for treating severe otitis externa in children and adolescents as it has been the subject of extensive investigation and is available in syrup form. ${ }^{10}$ 
Besides its systemic effect, ciprofloxacin is used more often topically for its local effects in the form of eye or ear drops. This fluoroquinolone thereby possesses a very broad spectrum of indications which range from complicated urinary tract infections, infections of the respiratory system, skin and bones, to severe typhoid salmonella infections, or bacterial conjunctivitis. Known side effects include gastrointestinal complaints (nausea, diarrhea, dyspepsia), disorders of the central nervous system (headache, nervousness/restlessness, dizziness, tremor, hyposmia), and skin irritations and eosinophilia. ${ }^{11}$

The undesirable effects of systemic treatment can be largely avoided through topical administration, however. A high local concentration is in fact attained, yet resorption does not occur. Therefore, itching or burning at the application site or superinfections of the ear can arise due to robust pathogens. Allergic reactions occur very rarely. ${ }^{10}$ Systemic side effects of local application occasionally include dizziness and headache.

\section{Materials and methods}

\section{Search methods used to identify studies}

The electronic databases Cochrane Ear, Nose and Throat Disorders Group Trials Register, The Cochrane Central Register of Controlled Trials (CENTRAL), PubMed, MEDLINE, EMBASE, and Web of Science were systematically searched for randomized, controlled studies. Using MeSH, a search term catalog was compiled with the following terms that were then entered in combinations: external ear, inflammation of the external ear, acute otitis externa, quinolone, ciprofloxacin $0.2 \%$, ciprofloxacin, solution, ear drops, drug therapy, anti-bacterial agents, antifungal, antibiotics.

Restrictions with respect to language, publication date, or publication status were not initially made. This review was also limited to published work. The last search was started on 1 March 2011.

\section{Patients}

Patients (both children and adults) with the diagnosis of acute otitis externa were included in the review. Not included were patients who suffered from a chronic form of external otitis or otitis media.

\section{Parameters}

Symptom improvement and microbiological eradication were defined as primary outcome parameters. Time to complete disappearance of symptoms and any side effects were observed as further aspects.

\section{Results}

\section{Search results}

Thirty-eight studies satisfied our search criteria, and we examined the abstracts of these. When this process was completed, the number of suitable studies decreased to 36 ; we then worked through their full texts (Figure 1). After three reviewers came to a consensus concerning further eliminations, six and eight studies, respectively, were available for this systematic review (Table 1). ${ }^{12-19}$

We were denied access to the full text of two of these eight randomized controlled trials. The comprehensive publication by Lildholdt et al ${ }^{15}$ and the text by Psifidis et a ${ }^{18}$ could not be requested, which is why detailed data are missing.

Unpublished studies were not considered in this systematic review.

\section{Background (included studies) Treatment doses}

Besides six studies that investigated a $0.2 \%$ ciprofloxacin drug, we also included two more studies that used a $0.3 \%$ ciprofloxacin product (Table 2). ${ }^{14,16}$

The ciprofloxacin dose of the ear solutions used in the individual studies was comparable. The majority planned a 7-day application phase, during which the study participants applied three drops to each ear twice daily. Marom et al ${ }^{16}$ raised the dose to four drops ( $0.3 \%$ ciprofloxacin), and Goldenberg et al ${ }^{14}$ doubled the application period to 2 weeks . The exact dose remains unclear in the study by Drehobl et a ${ }^{13}$ in which the study period was also 7 days, with applications twice daily, but the study specified the dose as ampoule ("vial") rather than stating the number of drops.

\section{Outcomes assessed}

\section{Clinical response}

Clinical success was, in part, classified differently and measured at various points in times (Table 2). In addition, the definition of treatment success varied slightly among the studies. Some studies defined clinical success as complete recovery (resolution) with complete freedom from symptoms. In others, it included mild symptoms, but a distinct improvement from the initial value. In principle, the symptoms and signs typical for the disease and used in the evaluation were similar. They included edemas, pain, or hypersensitivity of the ear, and otorrhea.

\section{Time to recovery - time to end of pain}

Time to end of pain constituted a relevant target value for three of the studies included: ${ }^{15,17,19}$ Pistorius et al ${ }^{17}$ determined 


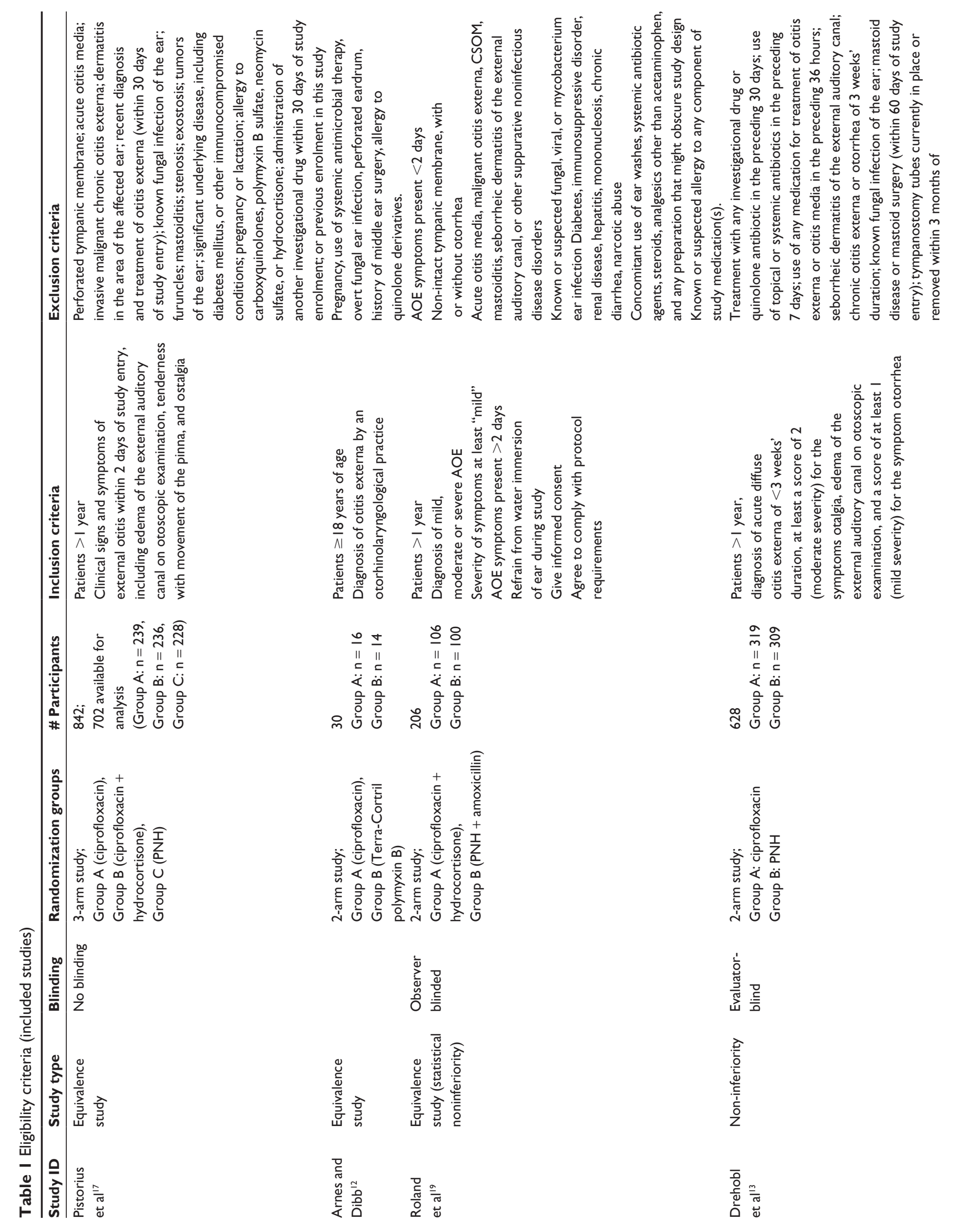



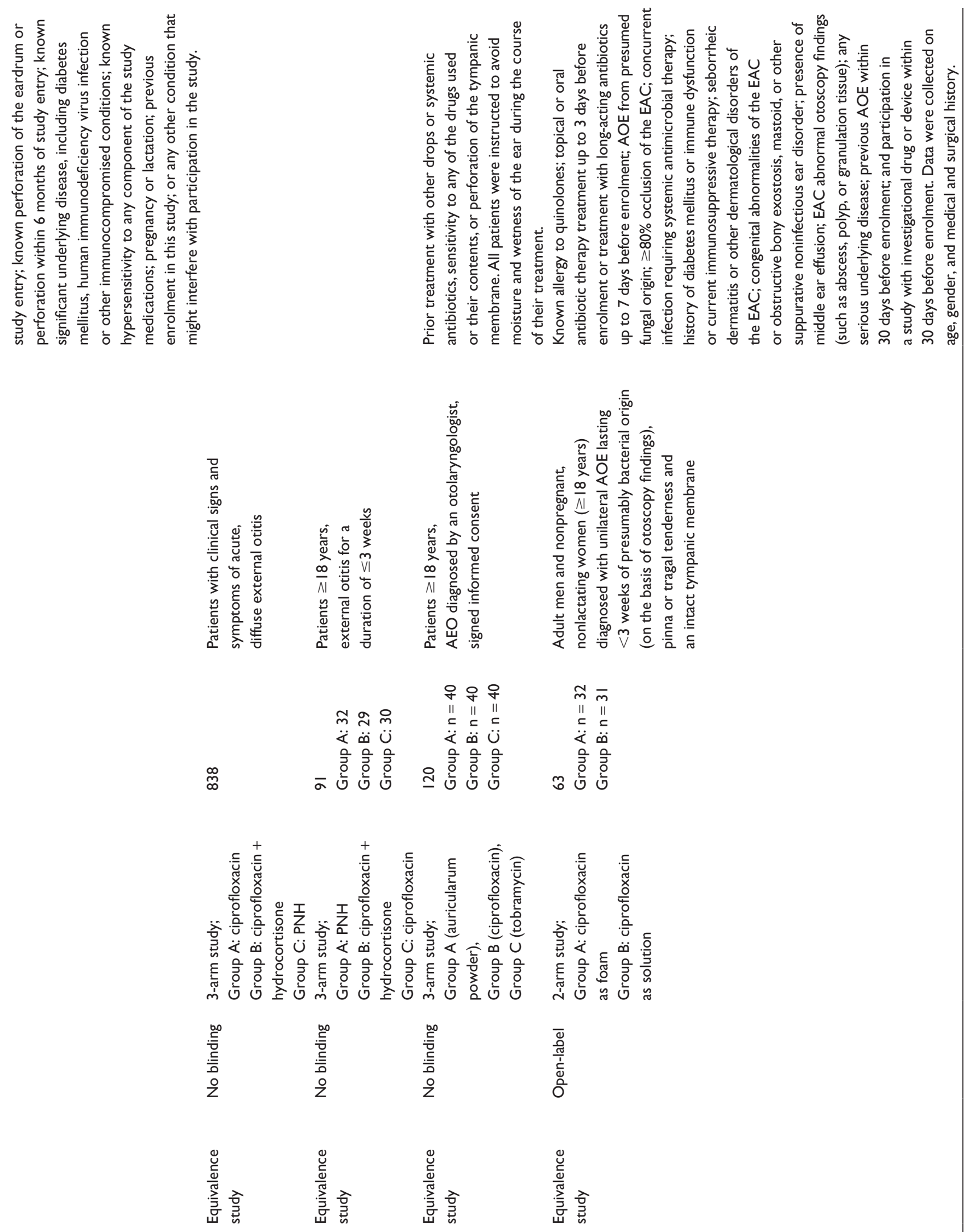

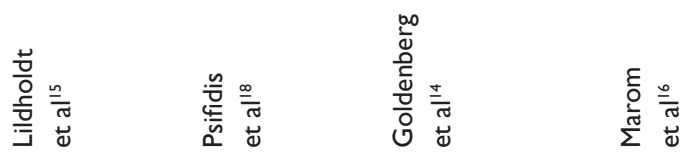


Table 2 Study design (included studies)

\begin{tabular}{|c|c|c|c|c|c|}
\hline Study ID & Medications & Duration and dose & Primary endpoint & Secondary parameters & Safety \\
\hline $\begin{array}{l}\text { Pistorius } \\
\text { et } \mathrm{al}^{17}\end{array}$ & $\begin{array}{l}\text { Group A: } \\
\text { Ciprofloxacin otic drops } \\
\text { as hydrochloride } \\
\text { monohydrate }(0.2 \%) \\
\text { Group B: } \\
\text { Ciprofloxacin otic drops } \\
\text { as hydrochloride } \\
\text { monohydrate }(0.2 \%) \text { plus } \\
\text { hydrocortisone }(0.1 \%) \\
\text { Group C: } \\
\text { combination of } \\
\text { Polymyxin B (I0.000 U), } \\
\text { neomycin sulfate }(3.5 \mathrm{mg} / \mathrm{mL}) \\
\text { and hydrocortisone }(0.1 \%)\end{array}$ & $\begin{array}{l}7 \text { days } \\
\text { Group A: } \\
3 \text { drops twice daily } \\
\text { Group B: } \\
3 \text { drops twice daily } \\
\text { Group C: } \\
3 \text { drops } 3 \text { times a day } \\
\text { ( }<13 \text { years of age) } \\
\text { or } 4 \text { drops } \\
\text { ( }>13 \text { years of age) }\end{array}$ & $\begin{array}{l}\text { Clinical success } \\
\text { (resolution or } \\
\text { improvement of } \\
\text { symptoms) at the } \\
\text { end of therapy } \\
\text { (Day 10-17) }\end{array}$ & $\begin{array}{l}\text { Antimicrobial effectiveness } \\
\text { (microbiological } \\
\text { eradication) at the end } \\
\text { of therapy (Day } 10-17 \text { ) } \\
\text { Time until ear pain } \\
\text { disappeared completely } \\
\text { via visual analog scale }\end{array}$ & $\begin{array}{l}\text { Medication- } \\
\text { related adverse } \\
\text { events }\end{array}$ \\
\hline $\begin{array}{l}\text { Arnes and } \\
\text { Dibb }^{12}\end{array}$ & $\begin{array}{l}\text { Group A: } \\
\text { Ciprofloxacin }(0.2 \%) \\
\text { as ear drops } \\
\text { Group B: } \\
\text { drops containing } \\
\text { oxytetracycline }(5 \mathrm{mg} / \mathrm{mL}) \\
\text { polymyxin B }(10,000 \text { units } / \mathrm{mL}) \\
\text { and hydrocortisone }(15 \mathrm{mg} / \mathrm{mL})\end{array}$ & $\begin{array}{l}7 \text { days } \\
\text { Group A: } \\
2-3 \text { drops twice daily } \\
\text { Group B: } \\
2-3 \text { drops twice daily }\end{array}$ & $\begin{array}{l}\text { Clinical success } \\
\text { (complete resolution, } \\
\text { marked improvement, } \\
\text { slight improvement, } \\
\text { failure, or } \\
\text { indeterminate) } \\
\text { at the end of } \\
\text { therapy (Day 8) }\end{array}$ & $\begin{array}{l}\text { Bacteriological assessment } \\
\text { (eradication, persistence, } \\
\text { recurrence, superinfection) } \\
\text { Individual (investigator's) } \\
\text { assessment (completely } \\
\text { successful, partially } \\
\text { successful, unsuccessful, } \\
\text { indeterminate) }\end{array}$ & $\begin{array}{l}\text { Clinical side } \\
\text { effects (adverse } \\
\text { events) }\end{array}$ \\
\hline $\begin{array}{l}\text { Roland } \\
\text { et } \text { al }^{19}\end{array}$ & $\begin{array}{l}\text { Group A: } \\
\text { Otic solution consisting } \\
\text { of ciprofloxacin and } \\
\text { hydrocortisone } \\
\text { Group B: } \\
\text { Combination of PNH } \\
\text { (polymyxin B/neomycin/ } \\
\text { hydrocortisone) } \\
\text { plus the antibiotic } \\
\text { amoxicillin }\end{array}$ & $\begin{array}{l}\text { Group A: } \\
7 \text { days, } \\
3 \text { drops twice daily } \\
\text { Group B: } \\
10 \text { days, } \\
2 \text { drops } 3 \text { times a day } \\
\text { ( }<17 \text { years of age) } \\
\text { or } 4 \text { drops } 3 \text { times a day } \\
\text { ( }>17 \text { years of age) }\end{array}$ & $\begin{array}{l}\text { Clinical success/ } \\
\text { response to therapy } \\
\text { (resolution) after } \\
\text { treatment ended } \\
\text { (Group A: Day 8, } \\
\text { Group B: Day II) }\end{array}$ & $\begin{array}{l}\text { Microbiological eradication } \\
\text { after treatment ended } \\
\text { (percentage of patients } \\
\text { with resolution of } \\
\text { disease-specific infection) } \\
\text { Time to end of pain } \\
\text { Symptom severity } \\
\text { (4-point scale for otalgia } \\
\text { and tenderness) }\end{array}$ & $\begin{array}{l}\text { Adverse events } \\
\text { or serious } \\
\text { adverse events }\end{array}$ \\
\hline $\begin{array}{l}\text { Drehobl } \\
\text { et } \mathrm{al}^{13}\end{array}$ & $\begin{array}{l}\text { Group A: } \\
\text { Cetraxal (ciprofloxacin otic } \\
\text { solution } 0.2 \% \text { ) } \\
\text { Group B: } \\
\text { PNH otic solution; } \\
\text { neomycin sulfate } \\
\text { ( } 3.5 \mathrm{mg} / \mathrm{mL} \text { neomycin base), } \\
\text { polymyxin B (I0 } 000 \mathrm{U}) \\
\text { and hydrocortisone }(1 \%)\end{array}$ & $\begin{array}{l}7 \text { days } \\
\text { Group A: } \\
\text { I vial twice daily } \\
\text { (morning and evening) } \\
\text { Group B: } \\
4 \text { drops } 3 \text { times daily } \\
\text { (for patients > } 13 \text { years } \\
\text { of age) or } 3 \text { drops } \\
\text { (for patients < } 13 \text { years } \\
\text { of age) (morning, } \\
\text { afternoon, evening) }\end{array}$ & $\begin{array}{l}\text { Clinical success } \\
\text { (proportion of } \\
\text { patients with clinical } \\
\text { cure) after follow-up } \\
\text { period (day I5-17). } \\
\text { Clinical cure was } \\
\text { defined as a score } \\
\text { of } 0 \text { for otalgia, } \\
\text { edema, and otorrhea }\end{array}$ & $\begin{array}{l}\text { Clinical success } \\
\text { (proportion of patients } \\
\text { with clinical cure) at the } \\
\text { end of treatment (Day 8-10) } \\
\text { Clinical improvement } \\
\text { (defined as a score of } 0 \\
\text { or I for otalgia, edema, } \\
\text { and otorrhea), resolution } \\
\text { of otalgia, and clinical + } \\
\text { microbiologic cure at the } \\
\text { end of treatment and after } \\
\text { the follow-up period }\end{array}$ & $\begin{array}{l}\text { Drug-related } \\
\text { adverse events }\end{array}$ \\
\hline $\begin{array}{l}\text { Lildholdt } \\
\text { et } \text { al }^{15}\end{array}$ & $\begin{array}{l}\text { Group A: } \\
\text { Ciprofloxacin }(0.2 \%) \\
\text { otic solution } \\
\text { Group B: } \\
\text { Combination of } \\
\text { ciprofloxacin }(0.2 \%) \\
\text { otic solution and } \\
\text { hydrocortisone }(0.1 \%) \\
\text { Group C: } \\
\text { Suspension of polymyxin } \\
\text { B-neomycin sulfate } \\
\text { (3.5 mg/mL)- } \\
\text { hydrocortisone (I\%) }\end{array}$ & $\begin{array}{l}7 \text { days } \\
\text { Group A: } \\
3 \text { drops twice daily } \\
\text { Group B: } \\
3 \text { drops twice daily } \\
\text { Group C: } \\
4 \text { drops } 3 \text { times a day }\end{array}$ & $\begin{array}{l}\text { Clinical success } \\
\text { (resolution or } \\
\text { improvement) } \\
\text { maintained at } \\
\text { follow-up about } \\
3 \text { weeks later }\end{array}$ & $\begin{array}{l}\text { Median time to end } \\
\text { of ear pain } \\
\text { Evaluation of ear cultures }\end{array}$ & \\
\hline
\end{tabular}

(Continued) 
Table 2 (Continued)

\begin{tabular}{|c|c|c|c|c|c|}
\hline Study ID & Medications & Duration and dose & Primary endpoint & Secondary parameters & Safety \\
\hline $\begin{array}{l}\text { Psifidis } \\
\text { et } \text { al }^{18}\end{array}$ & $\begin{array}{l}\text { Group A: } \\
\text { Combination of } \\
\text { polymyxin B ( } 10,000 \mathrm{U} / \mathrm{mL}) \text {, } \\
\text { neomycin }(3.5 \mathrm{mg} / \mathrm{mL}) \text {, } \\
\text { and hydrocortisone } \\
\text { (I0 mg/mL) } \\
\text { Group B: } \\
\text { Combination of } 0.2 \% \\
\text { ciprofloxacin }(2 \mathrm{mg} / \mathrm{mL}) \text { and } \\
\text { hydrocortisone }(10 \mathrm{mg} / \mathrm{mL}) \\
\text { Group C: } \\
\text { Ciprofloxacin }(0.2 \%) \text { alone }\end{array}$ & $\begin{array}{l}7 \text { days } \\
\text { Group A: } \\
3 \text { drops } 3 \text { times daily } \\
\text { Group B: } \\
3 \text { drops twice daily } \\
\text { Group C: } \\
3 \text { drops twice daily }\end{array}$ & $\begin{array}{l}\text { Clinical success } \\
\text { (complete } \\
\text { resolution of } \\
\text { external otitis) } \\
\text { at the end of the } \\
\text { follow-up period } \\
\text { (Day 2I-35) }\end{array}$ & $\begin{array}{l}\text { Microbiological } \\
\text { effectiveness (eradication, } \\
\text { persistence, superinfection) }\end{array}$ & Adverse events \\
\hline $\begin{array}{l}\text { Goldenberg } \\
\text { et } \mathrm{al}^{14}\end{array}$ & $\begin{array}{l}\text { Group A: } \\
\text { Auricularum powder } \\
\text { (dexamethasone } \\
10 \text { mg, oxytetracycline } \\
\mathrm{HCl} 90,000 \mathrm{U} \text {, polymyxin B } \\
\text { sulfate } 100,000 \mathrm{U} \text {, nystatin } \\
\text { I,000,000 U; Trima, Serolam } \\
\text { Laboratories, Germany) } \\
\text { Group B: } \\
\text { Ciprofloxacin 0.3\% } \\
\text { (Ciloxan, Alcon Laboratories, } \\
\text { Fort Worth, TX) } \\
\text { Group C: Tobramycin } \\
\text { (Tobrex, Alcon Laboratories) }\end{array}$ & $\begin{array}{l}\text { I4 days } \\
\text { Group A: } \\
\text { I application } \\
\text { twice daily } \\
\text { Group B: } \\
3 \text { times a day } \\
\text { Group C: } \\
3 \text { times a day }\end{array}$ & $\begin{array}{l}\text { Clinical success } \\
\text { (rate of cure) at } \\
\text { Day 3-4 after } \\
\text { initial treatment }\end{array}$ & $\begin{array}{l}\text { Clinical success } \\
\text { (rate of cure) at } \\
\text { Day I4 } \\
\text { Microbiological } \\
\text { effectiveness }\end{array}$ & Adverse events \\
\hline $\begin{array}{l}\text { Marom } \\
\text { et } \text { al }^{16}\end{array}$ & $\begin{array}{l}\text { Group A: } \\
\text { Foam Otic Cipro, } 0.3 \% \\
\text { ciprofloxacin } \\
\text { foam-based formulation } \\
\text { Group B: } \\
\text { Ciloxan, } 0.3 \% \\
\text { solution-based ciprofloxacin }\end{array}$ & $\begin{array}{l}7 \text { days } \\
\text { Group A: } \\
\text { One application } \\
\text { twice daily } \\
\text { Group B: } \\
4 \text { drops twice daily }\end{array}$ & $\begin{array}{l}\text { Clinical response/ } \\
\text { cure defined as } \\
\text { resolution (absence } \\
\text { of AOE-related signs } \\
\text { and symptoms) or } \\
\text { improvement } \\
\text { (presence of AOE- } \\
\text { related minor signs } \\
\text { or symptoms, with } \\
\text { no further therapy } \\
\text { required) at the end } \\
\text { of therapy (Day 8-14) }\end{array}$ & $\begin{array}{l}\text { Otorrhea cessation } \\
\text { Pain relief }\end{array}$ & Adverse events \\
\hline
\end{tabular}

Abbreviation: AOE, acute otis externa.

this parameter via the Visual Analog Scale; Lildholdt et al ${ }^{15}$ and Roland et $\mathrm{a}^{19}$ rated pain perception in diary entries on a scale of $0-4$.

Goldenberg et $\mathrm{al}^{14}$ evaluated pain intensity at two set times: Day 3-4 and Day 14.

An alternative approach to analyzing pain was chosen by Marom et $\mathrm{al}^{16}$ who, instead of the time to end of pain, evaluated the basic development of pain perception based on daily entries via the visual analog scale.

\section{Microbiological response}

With the exception of one study, the microbiological effectiveness of the study medication, among others, was measured as the secondary outcome measure. ${ }^{16}$
Upon inclusion of the patients, samples were taken to determine the causative organisms. This procedure was repeated at the end of treatment (or alternatively after a follow-up period). The classification scheme was defined differently in the individual studies:

Drehobl et al ${ }^{13}$ divided the samples into "no exudates observed", "exudate was present, but there was no growth on culture", "exudate was present, and culture showed some pathogen growth at baseline or patient's response was clinical failure", or "exudate was present, and culture showed one or more new pathogens" and assessed them at the end of treatment and also after a follow-up period. Patients who tested positive for bacteria at the beginning of the study and then tested negative later during the study came into the category "microbial cure." 
Table 3 Results

\begin{tabular}{|c|c|c|c|c|c|}
\hline \multirow[t]{2}{*}{ Study ID } & \multicolumn{2}{|l|}{ Clinical response } & \multirow{2}{*}{$\begin{array}{l}\text { Time to } \\
\text { end of pain }\end{array}$} & \multicolumn{2}{|c|}{ Microbiological response } \\
\hline & Definition & Numbers & & $\begin{array}{l}\text { Bacteriological } \\
\text { assessment }\end{array}$ & Effectiveness \\
\hline Pistorius et al $^{17}$ & $\begin{array}{l}\text { Clinical resolution } \\
\text { or improvement }\end{array}$ & $\begin{array}{l}\text { Group A: } 93 \% \\
\text { Group B: } 90 \% \\
\text { Group C: } 87 \%\end{array}$ & $\begin{array}{l}\text { Group A: } 4.7 \text { days } \\
\text { Group B: } 3.8 \text { days } \\
\text { Group C: } 4.1 \text { days }\end{array}$ & $\begin{array}{l}\text { Pseudomonas } \\
\text { aeruginosa: } 67 \%\end{array}$ & $\begin{array}{l}\text { Bacteriological } \\
\text { eradication } \\
\text { (including presumed } \\
\text { eradication) } \\
\text { Group A: } 92 \% \\
\text { Group B: } 95 \% \\
\text { Group C: } 87 \%\end{array}$ \\
\hline Arnes and Dibb ${ }^{12}$ & $\begin{array}{l}\text { Complete success, } \\
\text { partial success, } \\
\text { unsuccessful, } \\
\text { indeterminate }\end{array}$ & $\begin{array}{l}\text { Complete success } \\
\text { Group A: I } 4 \text { (87.5\%) } \\
\text { Group B: } 5 \text { (35.7\%) } \\
\text { Partial success } \\
\text { Group A: } 2 \text { (I2.5\%) } \\
\text { Group B: } 4 \text { (28.6\%) } \\
\text { Unsuccessful or } \\
\text { indeterminate } \\
\text { Group A: } 0 \\
\text { Group B: } 5 \text { (35.7\%) }\end{array}$ & & $\begin{array}{l}\text { P. aeruginosa } \\
\text { Group A: } 6 \text { (37.5\%) } \\
\text { Group B: } 7 \text { (50\%) }\end{array}$ & $\begin{array}{l}\text { Eradication } \\
\text { Group A: I5 (93.75\%) } \\
\text { Group B: } 7 \text { (50\%) } \\
\text { Persistence } \\
\text { Group A: I (6.25\%) } \\
\text { Group B: } 7 \text { (50\%) } \\
\text { Superinfection } \\
\text { Group A: I (6.25\%) } \\
\text { Group B: } 0\end{array}$ \\
\hline Roland et al ${ }^{19}$ & $\begin{array}{l}\text { Cured or } \\
\text { improved } \\
7 \text { days after } \\
\text { treatment ended }\end{array}$ & $\begin{array}{l}\text { Group A: } 94.3 \% \\
\text { Group B: } 89.8 \%\end{array}$ & $\begin{array}{l}\text { Group A: } 6 \text { days } \\
\text { Group B: } 6 \text { days }\end{array}$ & & $\begin{array}{l}\text { Eradication } \\
\text { Group A: } 67 \text { (95.7\%) } \\
\text { Group B: } 53 \text { (89.8\%) } \\
\text { Superinfection } \\
\text { Group A: I (I.4\%) } \\
\text { Group B: } 2(3.4 \%) \\
\text { Failure } \\
\text { Group A: } 2(2.9 \%) \\
\text { Group B: } 4(6.8 \%)\end{array}$ \\
\hline Drehobl et $\mathrm{al}^{13}$ & $\begin{array}{l}\text { Clinical cure of } \\
\text { otitis symptoms } \\
\text { after follow-up } \\
\text { (score } 0 \text { for } \\
\text { otalgia, edema, } \\
\text { and otorrhea) }\end{array}$ & $\begin{array}{l}\text { Group A. } 86.6 \% \\
\text { Group B: } 81.1 \%\end{array}$ & & & $\begin{array}{l}\text { P. aeruginosa } \\
\text { Group A: } 87.5 \% \\
\text { Group B: } 78.6 \% \\
\text { Staphylococcus aureus } \\
\text { Group A: } 72.7 \% \\
\text { Group B: } 75.9 \%\end{array}$ \\
\hline Lildholdt et a $\left.\right|^{15}$ & $\begin{array}{l}\text { Resolution or } \\
\text { improvement } \\
\text { after follow- } \\
\text { up period } \\
\text { ( } 3 \text { weeks later) }\end{array}$ & $\begin{array}{l}\text { Group A: } \sim 95 \% \\
\text { Group B: } \sim 95 \%\end{array}$ & $\begin{array}{l}\text { Median: } 4.8 \text { days } \\
\text { (no statistically } \\
\text { significant difference) }\end{array}$ & P. aeruginosa: $64 \%$ & $\begin{array}{l}\text { Persisting P. aeruginosa } \\
\text { Group A: } 9 \text { (8.7\%) } \\
\text { Group B: I I (9.4\%) } \\
\text { Group C: } 22(21.4 \%)\end{array}$ \\
\hline Psifidis et $\mathrm{al}^{18}$ & $\begin{array}{l}\text { Complete resolution } \\
\text { of external otitis }\end{array}$ & $\begin{array}{l}\text { Group A: } 84.4 \% \\
\text { Group B: } 100 \% \\
\text { Group C: } 96.7 \%\end{array}$ & & $\begin{array}{l}\text { P, aeruginosa } \\
\text { Group A: I } 8 \text { (56.3\%) } \\
\text { Group B: I } 3(44.8 \%) \\
\text { Group C: II (36.7\%) }\end{array}$ & $\begin{array}{l}\text { Eradication } \\
\text { Group A: } 72 \% \\
\text { Group B: } 83.3 \% \\
\text { Group C: } 93.8 \% \\
\text { Persistence } \\
\text { Group A: } 12 \% \\
\text { Group B: } 5.6 \% \\
\text { Group C: } 6.3 \% \\
\text { Superinfection } \\
\text { Group A: } 16 \% \\
\text { Group B: } 11.1 \% \\
\text { Group C: } 0 \%\end{array}$ \\
\hline Goldenberg et al ${ }^{14}$ & $\begin{array}{l}\text { Cured at day } \\
3-4 \text { after } \\
\text { initial treatment }\end{array}$ & $\begin{array}{l}\text { Group A: } 86 \% \\
\text { Group B: } 77 \% \\
\text { Group C: } 56 \%\end{array}$ & & $\begin{array}{l}\text { P.: } 86 \text { (72\%) } \\
\text { S. aureus: } 22 \text { (18\%) } \\
\text { Proteus mirabilis: } 6(5 \%) \\
\text { Coagulase-negative } \\
\text { Staphylococcus: } 6 \text { (5\%) }\end{array}$ & $\begin{array}{l}\text { P. aeruginosa } \\
\text { Group A: } 0 \\
\text { Group B: } 0 \\
\text { Group C: } 10 \%\end{array}$ \\
\hline
\end{tabular}


Table 3 (Continued)

\begin{tabular}{|c|c|c|c|c|c|}
\hline \multirow[t]{2}{*}{ Study ID } & \multicolumn{2}{|l|}{ Clinical response } & \multirow{2}{*}{$\begin{array}{l}\text { Time to } \\
\text { end of pain }\end{array}$} & \multicolumn{2}{|c|}{ Microbiological response } \\
\hline & Definition & Numbers & & $\begin{array}{l}\text { Bacteriological } \\
\text { assessment }\end{array}$ & Effectiveness \\
\hline & & & & & $\begin{array}{l}\text { S. aureus } \\
\text { Group A: } 0 \\
\text { Group B: } 2.5 \% \\
\text { Group C: } 0 \\
\text { Staphylococcus } \\
\text { coagulase-negative } \\
\text { Group A: } 0 \\
\text { Group B: } 5 \% \\
\text { Group C: } 10 \%\end{array}$ \\
\hline Marom et $\mathrm{al}^{16}$ & $\begin{array}{l}\text { Resolution (absence } \\
\text { of signs and symptoms) } \\
\text { or improvement (presence } \\
\text { of symptoms with no } \\
\text { further therapy required) }\end{array}$ & $\begin{array}{l}\text { I) PP population } \\
\text { Resolution } \\
\text { Group A: } 25 \text { (86.2\%) } \\
\text { Group B: } 22 \text { (78.6\%) } \\
\text { Improvement } \\
\text { Group A: } 4 \text { (13.8\%) } \\
\text { Group B: } 6 \text { (21.4\%) } \\
\text { 2) ITT population } \\
\text { Resolution + } \\
\text { improvement } \\
\text { Group A: } 93.6 \% \\
\text { Group B: } 93.8 \%\end{array}$ & & & \\
\hline
\end{tabular}

Abbreviations: ITT, intention-to-treat; PP; per-protocol.

Arnes et $\mathrm{al}^{12}$ Pistorius et $\mathrm{a} \mathrm{l}^{17}$, and Psifidis et $\mathrm{a} \mathrm{l}^{18}$ defined outcome critaria from "eradication" to "superinfection" or "reinfection" and examined the microbiological activity at the end of treatment and after follow-up. The change or the reduction of pathogenic infections could be determined in this way for each group and each pathogen.

In contrast, Lildholdt et $\mathrm{al}^{15}$ in their study evaluated the number of persisting cultures after the end of treatment, which was also relevant for Goldenberg et a ${ }^{14}$ that is, whether and to what extent bacterial proliferation still existed after therapy was completed.

\section{Adverse events}

In the majority of the studies, adverse events were evaluated as an expression of safety. Four studies specified such events additionally $13,16,17,19$ by explicitly analyzing medicationrelated adverse events; three studies evaluated clinical side effects/adverse events in general. ${ }^{12,14,18}$ Whether adverse events were of relevance in the study by Lildholdt et al remains unclear.

\section{Timing of outcome assessment}

The relevant point in time at which the primary and secondary outcome measures were assessed differed among the studies. Whereas in some studies the data were included in the analysis directly after the application phase was completed, others defined the time after a follow-up phase as decisive for the analysis. In only one case, the data from Day 3-4 formed the basis of the analysis. ${ }^{14}$

In the studies that conducted an analysis with data directly after treatment had ended, the point in time varied between Day 8 and Day 17. In the studies that collected data relevant to the target value after a follow-up phase, the time span ranged from Day 15 to Day 35.

\section{Study results}

\section{Clinical cure}

The included studies demonstrate the statistical equivalence between ciprofloxacin $(0.2 \%)$ and the reference product PNH (Table 3). Some studies investigated the cure rate after completion of treatment. ${ }^{12,17,19}$ Here, the rate for patients whose condition fell into the category "clinical resolution" or "improvement" ranged between $93 \%$ and $100 \%$. Studies that evaluated the outcome parameters after a follow-up period showed cure or improvement rates between $86.6 \%$ and $96.7 \%{ }^{13,15,18}$ Consequently, comparably high success rates for the ciprofloxacin $0.2 \%$ drug were determined in these studies.

Similar results were ascertained in the studies that investigated a higher concentration of ciprofloxacin $(0.3 \%) .^{14,16} \mathrm{In}$ 
Table 4 Adverse events

\begin{tabular}{|c|c|c|c|c|}
\hline Study ID & Adverse events & Medication-related AE & Type and severity & $\begin{array}{l}\text { Premature } \\
\text { discontinuation }\end{array}$ \\
\hline Pistorius et al ${ }^{17}$ & $\begin{array}{l}\text { Group A: } 66 \\
(23 \%) \\
\text { Group B: } 70 \\
(25 \%) \\
\text { Group C: } 55 \\
(20 \%)\end{array}$ & $\begin{array}{l}\text { Group A: } 6 \% \\
\text { Group B: } 5 \% \\
\text { Group C: } 5 \%\end{array}$ & $\begin{array}{l}\text { Headache, ear pain, } \\
\text { pruritus mainly mild or } \\
\text { moderate in severity }\end{array}$ & $\begin{array}{l}\text { Group A: I } \\
\text { Group B: } 4 \\
\text { Group C: } 3\end{array}$ \\
\hline Arnes and Dibb ${ }^{12}$ & None & & & \\
\hline Roland et al ${ }^{19}$ & $\begin{array}{l}\text { Group A: } 6 \\
(5.7 \%) \\
\text { Group B: } 5 \\
(5 \%)\end{array}$ & $\begin{array}{l}\text { Group A: } 0 \\
\text { Group B: I } \\
(1.0 \%)\end{array}$ & $\begin{array}{l}\text { Mostly not serious } \\
\text { (I breast cancer) }\end{array}$ & $\begin{array}{l}\text { I I (in most cases } \\
\text { otitis media) }\end{array}$ \\
\hline Drehobl et $\mathrm{al}^{13}$ & & $\begin{array}{l}\text { Group A: I I } \\
(3.8 \%) \\
\text { Group B: I I } \\
(3.6 \%)\end{array}$ & $\begin{array}{l}\text { Ear pruritus, headache, } \\
\text { ear discomfort, application } \\
\text { site pain/burning mostly } \\
\text { of mild intensity }\end{array}$ & $\begin{array}{l}\text { Group A: } 3 \\
\text { Group B: } 3\end{array}$ \\
\hline $\begin{array}{l}\text { Lildholdt et } \text { al }^{15} \\
\text { Psifidis et } \text { al }^{18} \\
\text { Goldenberg et } \text { al }^{14}\end{array}$ & $\begin{array}{l}\text { None } \\
\text { None }\end{array}$ & & & \\
\hline Marom et $\mathrm{al}^{16}$ & $\begin{array}{l}\text { Group A: } 7 \\
(21 \%) \\
\text { Group B: } 5 \\
(16 \%)\end{array}$ & $\begin{array}{l}\text { Group A: } 4 \\
(12 \%)+\text { I serious AE } \\
\text { Group B: I }(3 \%)\end{array}$ & $\begin{array}{l}\text { Otalgia, tinnitus, pruritus, } \\
\text { diarrhea, headache, } \\
\text { throat pain }\end{array}$ & $\begin{array}{l}\text { Group A: I } \\
\text { Group B: } 0\end{array}$ \\
\hline
\end{tabular}

the study by Goldenberg et al ${ }^{14}$ about $77 \%$ of patients who were treated with ciprofloxacin fulfilled the definition of cure on Day 3-4. After 14 days, the rate reached 100\%. Marom et $\mathrm{al}^{16}$ found study participants in the per-protocol population to be $100 \%$ symptom-free at the end of a 7-day treatment phase. In the intent-to-treat population, complete resolution

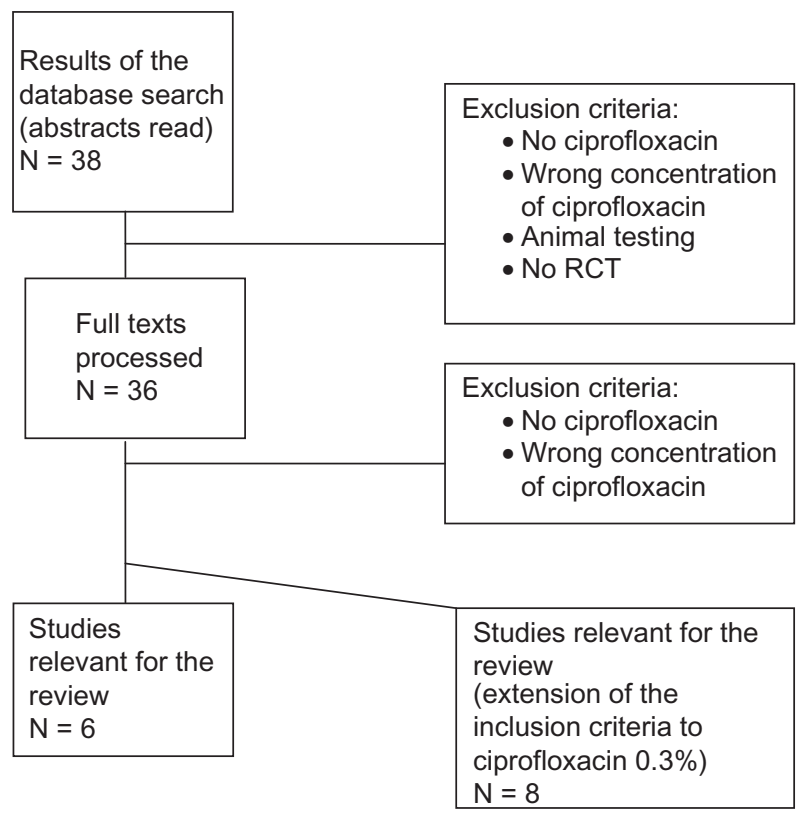

Figure I Flow chart. was observed in $93.6 \%$ of the patients in the ciprofloxacin group at the same point in time.

\section{Microbial cure}

The authors consistently identified high in vitro activity of ciprofloxacin against $P$. aeruginosa with high eradication rates of $83.3 \%$ to $95.7 \%$ and rare cases of persisting organisms or superinfections (Table 3 ).

Psifidis et $\mathrm{al}^{18}$ and Pistorius et $\mathrm{al}^{17}$ who, besides ciprofloxacin $0.2 \%$, also tested a combination of ciprofloxacin $0.2 \%$ and hydrocortisone $0.1 \%$, observed that the addition of hydrocortisone raised the eradication rate even further.

In the treatment of patients who had an infection with S. aureus bacteria, ciprofloxacin proved effective in $72.7 \%$ of patients.

\section{Adverse events}

No adverse events occurred in some studies, ${ }^{12,18,19}$ but in others, incidents that could be attributed to the medication took place at a rate of $3 \%-6 \%$ in the groups treated with ciprofloxacin (Table 4). The majority of studies spoke exclusively of mild side-effects, with similar frequencies in the individual groups; premature discontinuation was rarely reported. Drehobl et $\mathrm{al}^{13}$ and Pistorius et $\mathrm{al}^{17}$ name headache, earache, and itching at the site of application as the main symptoms that could be linked to the trial medication. 


\section{Risk of bias}

The greatest susceptibility to systematic distortions of the study results constituted the insufficient blinding of the included studies. While two study groups explicitly mentioned using non-blinding, ${ }^{16,18}$ four other authors made no comment whatsoever in this regard. ${ }^{12,14,15,18}$ Based on the fact that blinding was not addressed, however, it is to be assumed that blinding did not occur and the studies were open-label. In the study by Drehobl et $\mathrm{al}^{13}$ the evaluator at least was blinded, and only Roland et a $1^{19}$ conducted an observer/investigatorblinded study.

In addition, the randomization procedure remained unclear in a large proportion of the studies. Although all were randomized, controlled studies according to the publications, the randomization process was mentioned in only three studies. ${ }^{12,16,19}$

Another deficit with reference to the included studies was the absence of two full texts. We could only draw on the information from the abstracts by Lildholdt et a ${ }^{15}$ and Psifidis et a $\mathrm{l}^{18}$ because we were denied access to the complete, comprehensive material.

\section{Discussion}

The outcome measure "clinical success" consistently shows higher success rates in patients treated with the fluoroquinolone, than in the control groups. At the same time, the authors point out - in addition to the effectiveness of the active ingredient - the absence of any ototoxicity and the low systemic exposure caused by ciprofloxacin.

In summary, clinical equivalence can be determined for both of the treatment possibilities ciprofloxacin/ hydrocortisone and PNH plus amoxicillin in adults and children. However, low systemic exposure, the absence of ototoxicity, and the smaller dose speak clearly for treatment with ciprofloxacin.

Ciprofloxacin stands out due to its low rate of side effects. Adequate safety is thereby given with this fluoroquinolone.

For this reason, ciprofloxacin is not only non-inferior to other classes of antibiotics but also to antibiotic drugs that are combined with glucocorticoids.

Studies that evaluated microbiological activity come to the conclusion that the organism P. aeruginosa represents the main pathogen in the investigated population having acute otitis externa. The authors consistently ascertained high in vitro activity of ciprofloxacin against $P$. aeruginosa.

Special attention must be paid to patient compliance in the included studies. The lower the required application rate of the otologic drug, the more probable it is that the patients adhere to therapy and apply the medicine regularly. Thus we can conclude that patients requiring fewer daily drug administrations will comply more closely with the treatment plan.

When considering the bias, and consequently the results, we should pay particular attention to a certain distortion: due to the different daily application rates of the otologic drugs used, double-blinding of the study could not always be achieved.

The possibility of including a much larger number of clinical studies in this review would have existed if the question at hand had also applied to ciprofloxacin $0.3 \%$ solution.

Other studies not included in this review combined ciprofloxacin $0.2 \%$ solution with the glucosteroid dexamethasone, which can be categorized as a glucocorticoid belonging to the active substance class two to three analogous to the classification of therapeutic index of topical dermatotherapy, and is thus to be considered potent. ${ }^{20}$

These combination drugs were in turn tested against "conventional" combination drugs such as PNH. The efficacy of ciprofloxacin could be therefore increased.

\section{Conclusion}

The studies included in this review demonstrate the statistical equivalence of ciprofloxacin $(0.2 \%)$ and the reference product $\mathrm{PNH}$, and thereby confirm the hypothesis of non-inferiority in terms of the cure rate and microbiological eradication. The efficacy of ciprofloxacin $0.2 \%$ antibiotic ear solution can be acknowledged.

\section{Disclosure}

The authors report no conflicts of interest.

\section{References}

1. Neher A, Nag1 M, Scholtz AW. Otitis externa: etiology, diagnostic and therapy. Hno. 2008;56(10):1067-1079.

2. Hajioff D, Mackeith S. Otitis externa. Clin Evid. 2010. pii: 0510.

3. Kaushik T, Malik T, Saeed S. Interventions for a acute otits externa. Cochrane Database Syst Rev. 2010;(1):CD004740.

4. Brook I. Microbiological studies of the bacterial flora of the external auditory canal in children. Acta Otolaryngol. 1981;91(3-4):285-287.

5. Berghaus A, Rettinger G, Böhme G. Hals-Nasen-Ohren-Heilkunde. Stuttgart: Hippokrates Verlag GmbH, 1996.

6. Osguthorpe JD, Nielsen DR. Otitis externa: review and clinical update. Am Fam Physician. 2006;74(9):1510-1516.

7. Sander R. Otitis externa: a practical guide to treatment and prevention. Am Fam Physician. 2001;63(5):927-936.

8. Scholz H, Schwabe U, (Hrsg.). Taschenbuch der arzneibehandlung angewandte pharmakologie. Berlin Heidelberg: Springer Verlag; 2005.

9. Lemmer B, Brune K, (Hrsg.). Pharmakotherapie - klinische pharmakologie. Heidelberg: Springer Medizin Verlag; 2007. 
10. AWMF. Leitlinie: Antibiotikatherapie der Infektionen an Kopf und Hals. Leitlinien der Deutschen Gesellschaft für Hals-Nasen-Ohren-Heilkunde, Kopf-und Hals-Chirurgie; 2008.

11. Shah PM. Ciprofloxacin. Int J Antimicrob Agents. 1991;1(2-3): 75-96.

12. Arnes E, Dibb WL. Otitis externa: clinical comparison of local ciprofloxacin versus local oxytetracycline, polymyxin B, hydrocortisone combination treatment. Curr Med Res Opin. 1993;13(3):182-186.

13. Drehobl M, Guerrero JL, Lacarte PR, Goldstein G, Mata FS, Luber S. Comparison of efficacy and safety of ciprofloxacin otic solution $0.2 \%$ versus polymyxin B-neomycin-hydrocortisone in the treatment of acute diffuse otitis externa*. Curr Med Res Opin. 2008;24(12): 3531-3542.

14. Goldenberg D, Golz A, Netzer A, Joachims HZ. The use of otic powder in the treatment of acute external otitis. Am J Otolaryngol. 2002;23(3): $142-147$.

15. Lildholdt T, Gehanno P, Kehrl W, Leiberman A. Otitis externa treated by topical antibiotics. Otolaryngol Head Neck Surg. 1997; 117(2):P116.
16. Marom T, Yelin R, Goldfarb A, et al. Comparison of safety and efficacy of foam-based versus solution-based ciprofloxacin for acute otitis externa. Otolaryngol Head Neck Surg. 2010;143(4):492-499.

17. Pistorius B, Westberry K, Drehobl M, et al; Otitis Externa Study Group. Prospective, randomized, comparative trial of ciprofloxacin otic drops, with or without hydrocortisone, vs polymyxin B-neomycinhydrocortisone otic suspension in the treatment of acute diffuse otitis externa. Infect Dis Clin Pract. 1999:8(8):387-395.

18. Psifidis A, Nikolaidis $\mathrm{P}, \mathrm{T}$ sona A, et al. The efficacy and safety of local ciprofloxacin in patients with external otitis: a randomized comparative study. Mediterranean Journal of Otology. 2005;1(1):20-23.

19. Roland PS, Belcher BP, Bettis R, et al; Cipro Study Group. A single topical agent is clinically equivalent to the combination of topical and oral antibiotic treatment for otitis externa. Am J Otolaryngol. 2008; 29(4):255-261.

20. Neurodermitis, Kontakekzem. Stiftung Warentest. http://www.test.de/ themen/gesundheit-kosmetik/medikamente/vom_arzt/a_haut_haare/a_ ekzem_neurodermitis/a_ekzem_neurodermitis/bespr.med/. Accessed April 25, 2011.
Therapeutics and Clinical Risk Management

\section{Publish your work in this journal}

Therapeutics and Clinical Risk Management is an international, peerreviewed journal of clinical therapeutics and risk management, focusing on concise rapid reporting of clinical studies in all therapeutic areas, outcomes, safety, and programs for the effective, safe, and sustained use of medicines. This journal is indexed on PubMed Central, CAS,

\section{Dovepress}

EMBase, Scopus and the Elsevier Bibliographic databases. The manuscript management system is completely online and includes a very quick and fair peer-review system, which is all easy to use. Visit http://www.dovepress.com/testimonials.php to read real quotes from published authors.

Submit your manuscript here: http://www.dovepress.com/therapeutics-and-clinical-risk-management-journal 